\title{
PARISAR: Patch-based estimation and regularized inversion for multi-baseline SAR interferometry
}

\author{
Giampaolo Ferraioli, Charles-Alban Deledalle, Loic Denis, Florence Tupin
}

\begin{abstract}
Reconstruction of elevation maps from a collection of SAR images obtained in interferometric configuration is a challenging task. Reconstruction methods must overcome two difficulties: the strong interferometric noise that contaminates the data, and the $2 \pi$ phase ambiguities. Interferometric noise requires some form of smoothing among pixels of identical height. Phase ambiguities can be solved, up to a point, by combining linkage to the neighbors and a global optimization strategy to prevent from being trapped in local minima. This paper introduces a reconstruction method, PARISAR, that achieves both a resolution-preserving denoising and a robust phase unwrapping by combining non-local denoising methods based on patch similarities and total-variation regularization. The optimization algorithm, based on graph-cuts, identifies the global optimum. Combining patch-based speckle reduction methods and regularization-based phase unwrapping requires solving several issues: (i) computational complexity, the inclusion of non-local neighborhoods strongly increasing the number of terms involved during the regularization, and (ii) adaptation to varying neighborhoods, patch comparison leading to large neighborhoods in homogeneous regions and much sparser neighborhoods in some geometrical structures. PARISAR solves both issues. We compare PARISAR with other reconstruction methods both on numerical simulations and satellite images and show a qualitative and quantitative improvement over state-of-the-art reconstruction methods for multi-baseline SAR interferometry.
\end{abstract}

Index Terms-SAR interferometry, multi-channel InSAR, Nonlocal means, TV regularization

\section{INTRODUCTION}

Phase unwrapping (PhU) operation is one of the most challenging tasks when reconstructing the height of earth surface based on Interferometric Synthetic Aperture Radar imaging [1]. PhU consists of retrieving the absolute value of the phase, starting from the $2 \pi$-wrapped data. Thanks to the widely known relation between the measured interferometric phase and the height of the observed scene [2], it is possible after adequate calibration steps and a PhU operation to recover the height of the observed area.

Several $\mathrm{PhU}$ algorithms have been developed in the last twenty years, and they can be classified into two main families: path-following methods and global optimization methods.

G. Ferraioli is with Dipartimento di Scienze e Tecnologie Università degli Studi di Napoli "Parthenope" - Naples, Italy

C.A. Deledalle is with CNRS-Univ. Bordeaux, IMB - Talence, France

L. Denis is with Univ Lyon, UJM-Saint-Etienne, CNRS, Institut d Optique Graduate School, Laboratoire Hubert Curien UMR 5516, F-42023, SAINTETIENNE, France

F. Tupin is with LTCI, Télécom ParisTech, Université Paris-Saclay, Paris, France

This work has been partially funded by ANR (the French National Research Agency) and DGA (Direction Générale de l'Armement) under ALYS project ANR-15-ASTR-0002.

Manuscript received XXX; revised XXX
Path-following PhU algorithms follow a path in the wrapped phase and unwrap each pixel locally. Algorithms from the second family minimize some measure of misfit between the unwrapped solution and wrapped one while promoting unwrapped solutions with few discontinuities. A good review of these algorithms can be found in [3] and [4].

Two difficulties make PhU a non-trivial operation: the first is due to the perturbations of interferometric noise on the acquired data; the second is the presence of phase differences larger than $\pi$ between two neighboring pixels, violating the so-called Itoh condition [5]. Such large phase differences arise when neighboring pixels have very different height values (i.e. in presence of discontinuities), or due to (strong) interferometric noise. Most existing algorithms account for the statistics of interferometric noise. The violation of Itoh condition makes the $\mathrm{PhU}$ problem ill-posed, thus challenging to solve. Commonly, to regularize the $\mathrm{PhU}$ problem and obtain a unique solution, differences between neighboring absolute phases are supposed to be less than $\pi$. This hypothesis is satisfied in the case of height profiles without strong discontinuities and high slopes, and for small baseline values [1].

$\mathrm{PhU}$ can be applied to more complex scenes with strong discontinuities or steep slopes by increasing the number of interferograms used during the inversion. By correctly combining different available interferograms, it is possible to restore the solution uniqueness without imposing constraints on the phase difference between neighboring pixels [6]. Multiple interferograms, commonly known as multi-channel interferograms, can be obtained in two different ways: using sensors working at different frequencies or using sensors acquiring the scene with different baselines. The latter, multi-baseline interferometry, is the case when the sensor observes the same scene, repeatedly, from slightly different positions, and is commonly the adopted one [7].

In the past years, multi-baseline $\mathrm{PhU}$ techniques have been largely investigated for height reconstruction [8], [9], [10], [11] and also for deformation retrieval applications (i.e. Differential Interferometry) [12][13]. More recently, new multibaseline height reconstruction algorithms have been proposed. A technique based on the extension of cluster analysis has been proposed in [14]. The reduction of memory requirements when dealing with multiple data is the main aim of [15]. The use of Kalman Filter in case of multiple acquisitions has been investigated in [16]. Finally, multi-baseline interferograms have also been used together with other information to improve reconstruction accuracy in urban areas: in [17] multibaseline data have been jointly processed with multi-aspect data while in [18] multi-baseline interferograms have been 
exploited together with amplitude information.

In order to obtain satisfying results using multi-baseline data, the first step is to correctly combine the available information. An effective way to combine the available multichannel (i.e., multi-baseline) interferometric data is to exploit statistical estimation methods. These methods propose to exploit the statistical distribution of the acquired data and to implement instruments provided by both classical [19], [20] and Bayesian estimation theory. In particular, for the latter when Markov Random Fields (MRF) theory is used for modeling the unknown height profile the so-called Bayesian Markovian estimation framework arises [21], providing very effective results in the multi-channel case [22], [23]. Interesting previous works proposed to apply the Bayesian Markovian framework to single-channel interferograms [24], [3].

In this paper we propose to exploit contextual information to improve multi-baseline unwrapping. Patch-based approaches, like NL-SAR [25], can effectively exploit local structural information in the noisy signal to gather similar samples and improve the estimation. To do so, they compare small pieces of information (the patches) and combine the similar ones. These estimators produce results with non-stationary residual variance: in regions where many similar patches are found, the estimate is accurate, while rare configurations are left almost unchanged, i.e., with the strong original interferometric variance. At these locations, an additional smoothing is to be enforced. Moreover, ambiguities due to phase wrapping can often be solved based on local smoothness priors. Markovian prior models of the elevation can be defined in this regard: total variation (TV) or truncated quadratic functions lead to smooth elevation while allowing strong discontinuities [18]. These regularization models applied alone suffer some limits like staircasing effects affecting low slope areas and leading to piecewise constant reconstruction [26]. Following the approach proposed in [27] for image and video, we investigate the combination of both a patch-based approach and TV regularization for elevation estimation in a multi-baseline interferometric framework, exploiting the whole statistical distribution of the interferometric data.

Contributions: The paper describes a strategy to perform this combination of non-local (i.e., patch-based) estimation and non-convex optimization. There are several possible ways to modify a regularization method in order to include nonlocal similarities. We show that, by using the weighted loglikelihood to account for these similarities, the complexity of the regularization step is left unchanged, which is an important aspect regarding the applicability of the method. Another key element of the proposed method is to account for the spatially variant standard deviation of the output of non-local speckle reduction methods. Regularization thus applies more strongly to regions with larger residual noise.

Section II describes the proposed model: the weighted loglikelihood term including patch-based similarities is introduced, then the TV regularization term and the global energy to be minimized, as well as the adopted optimization scheme are presented. In section III, an in-depth study of the proposed model is performed through experiments on simulated data, while results on real images are presented and discussed in section IV.

\section{THE MODEL}

A multi-channel interferogram with $D$ channels is formed by the collection, for each pixel $i$, of the $D$-dimensional complex-valued scattering vector $\boldsymbol{g}_{i}$. Under the classical hypothesis of fully developed speckle (Goodman model [28]), the scattering vector $\boldsymbol{g}_{i}$ is distributed according to a circular complex Gaussian:

$$
\mathrm{p}\left(\boldsymbol{g}_{i} \mid \boldsymbol{\Sigma}_{i}\right)=\frac{1}{\pi^{D} \operatorname{det}\left(\boldsymbol{\Sigma}_{i}\right)} \exp \left(-\boldsymbol{g}_{i}^{\dagger} \boldsymbol{\Sigma}_{i}^{-1} \boldsymbol{g}_{i}\right)
$$

with $\boldsymbol{g}_{i}^{\dagger}$ the Hermitian transpose of the column vector $\boldsymbol{g}_{i}$. This distribution is parameterized by the $D \times D$ complex covariance matrix $\boldsymbol{\Sigma}_{i}=\mathbb{E}\left[\boldsymbol{g}_{i} \boldsymbol{g}_{i}^{\dagger}\right]$ ( $\mathbb{E}$ denoting the expectation) at pixel $i$. This covariance matrix can be decomposed as:

$$
\boldsymbol{\Sigma}_{i}=\boldsymbol{R}_{i}^{1 / 2} \cdot \boldsymbol{\Gamma}_{i} \cdot \boldsymbol{R}_{i}^{1 / 2}
$$

where $\boldsymbol{R}_{i}$ is a diagonal matrix with $\left[\boldsymbol{R}_{i}\right]_{a, a}=r_{a}=$ $\mathbb{E}\left\{\left|\left[\boldsymbol{g}_{i}\right]_{a}\right|^{2}\right\}$ the reflectivity at pixel $i$ in channel $a$, and $\boldsymbol{\Gamma}_{i}$ is the coherence matrix given by

$$
\boldsymbol{\Gamma}_{i}=\left(\begin{array}{cccc}
1 & s_{1,2} & \cdots & s_{1, D} \\
s_{1,2}^{*} & 1 & & s_{2, D} \\
\vdots & & \ddots & \vdots \\
s_{1, D}^{*} & s_{2, D}^{*} & & 1
\end{array}\right)
$$

with $s_{a, b}=\mathbb{E}\left\{\left[\boldsymbol{g}_{i}\right]_{a} \cdot\left[\boldsymbol{g}_{i}\right]_{b}^{*}\right\} / \sqrt{r_{a} r_{b}}=\gamma_{a, b} \exp \left(j \psi_{a, b}\right)$ the inter-channel complex coherence between channels $a$ and $b$, $\gamma_{a, b}$ the coherence and $\psi_{a, b}$ the interferometric phase.

Provided that the images have been properly pre-processed in order to correct for flat earth and atmospheric phase distortions, the interferometric phases $\psi_{a, b}$ are related to the height $h$ through a function $f_{a, b}$ that accounts for the interferometric baselines [1]:

$$
\psi_{a, b}=f_{a, b}(h)=\alpha_{a, b} \cdot h=\frac{4 \pi B_{\perp}(a, b)}{\lambda \rho_{0} \sin \theta} h,
$$

where $\lambda$ is the working wavelength, $B_{\perp}(a, b)$ is the orthogonal baseline between channels $a$ and $b, \rho_{0}$ is the distance to the scene, and $\theta$ is the view angle.

In multi-baseline interferometry, a first step generally consists of estimating the covariance matrix $\boldsymbol{\Sigma}_{i}$ at pixel $i$ by spatial averaging over a square window $\mathcal{W}_{i}$ centered on $i$ :

$$
\widehat{\boldsymbol{\Sigma}}_{i}^{\text {(box) }}=\frac{1}{N} \sum_{j \in \mathcal{W}_{i}} \boldsymbol{g}_{j} \boldsymbol{g}_{j}^{\dagger} .
$$

$N$ being the number of samples in $\mathcal{W}_{i}$. The phases $\hat{\psi}_{a, b}$ extracted from this empirical covariance matrix are then inverted, in a second step, to produce an estimate $\hat{h}$ of the height such that $\hat{\psi}_{a, b} \approx f_{a, b}(\hat{h})$ for all channels $a$ and $b$.

Such an approach suffers from two drawbacks: (i) the first step involves an averaging procedure that degrades the spatial resolution by blurring thin structures, and (ii) the height estimation does not consider estimated heights at neighboring locations, thereby producing very noisy estimates in low coherence regions. 
In order to address these drawbacks, we propose to follow a Maximum a Posteriori (MAP) approach. In Bayesian estimation theory, a MAP estimator is computed by minimizing the a posteriori energy $\mathcal{E}$, which is the sum of two terms: the (neg)-log-likelihood term (aka "data term" $\mathcal{D}$ ) and the $a$ priori term (aka "regularization"). The bias and variance of the estimator are controlled by balancing the relative weight of those two terms. Given the strong fluctuations of point estimates of interferometric phase, we consider in paragraph II-A a generalization of the log-likelihood term to include a form of averaging over similar pixels within an extended neighborhood. The smoothing enforced by the a priori term to produce a satisfying estimate has then no need to be as severe as for a point estimate. We discuss the definition of the a priori term in paragraph II-B.

\section{A. Weighted log-likelihood term}

The statistical model defined by Eq.(1) leads to the following log-likelihood term at pixel $i$ (with const. a constant term):

$$
-\log \mathrm{p}\left(\boldsymbol{g}_{i} \mid \boldsymbol{\Sigma}_{i}\right)=\log \operatorname{det}\left(\boldsymbol{\Sigma}_{i}\right)+\boldsymbol{g}_{i}^{\dagger} \boldsymbol{\Sigma}_{i}^{-1} \boldsymbol{g}_{i}+\text { const. }
$$

The number of unknowns in $\boldsymbol{\Sigma}_{i}$ is larger than the number $D$ of observations in $\boldsymbol{g}_{i}$. Estimation of $h_{i}$ alongside of $\boldsymbol{R}_{i}$ and $\gamma_{a, b}$ values with a MAP estimator would thus rely on the choice of regularization terms expressed on all these unknowns. Designing such a regularization may be difficult due to the different nature of the unknowns: radiometry, coherence, and height, and their non-linear interaction in the definition of $\boldsymbol{\Sigma}_{i}$ in Eq.(2). To circumvent these problems, we choose to replace the log-likelihood term of covariance matrix $\boldsymbol{\Sigma}_{i}$ with a more general expression: the weighted loglikelihood [29], [30], [31]. This term considers not only the scattering vector $\boldsymbol{g}_{i}$ but all $\boldsymbol{g}_{j}$, for $j$ spanning all pixel indices of an extended ${ }^{1}$ neighborhood $\mathcal{N}_{i}$ centered on pixel $i$, as:

$$
\mathcal{D}_{i}=-\sum_{j \in \mathcal{N}_{i}} \omega_{i, j} \log \mathrm{p}\left(\boldsymbol{g}_{j} \mid \boldsymbol{\Sigma}_{i}\right)
$$

with $\omega_{i, j}$ a weight given to $\boldsymbol{g}_{j}$ in the estimation at pixel $i$. Such weights are typically chosen in a data-driven way in order to select only samples that are relevant for the subsequent estimation. In words, the covariance $\boldsymbol{\Sigma}_{i}$ is not only required to support the observation at pixel $i$ but also observations at all the pixels $j$ for which the weights $\omega_{i, j}$ are large. Minimizing (7) while setting the weights $\omega_{i, j}$ to be equal to each other within the square window $\mathcal{W}_{i}$ centered on $i$ and equal to 0 outside leads to Eq.(5), i.e., the boxcar covariance estimator. Spatially extending the number of observations related to a given covariance matrix $\boldsymbol{\Sigma}_{i}$ reduces the need for a regularization since the number of unknowns becomes much smaller than the number of observations. This however comes at a price: by mixing observations from different spatial locations $j$ in the estimation of $\boldsymbol{\Sigma}_{i}$, the spatial resolution is reduced. It is therefore crucial that the weights $\omega_{i, j}$ be carefully chosen so

\footnotetext{
${ }^{1}$ while the window used for boxcar filtering is typically smaller than $7 \times 7$ or $9 \times 9$, the extended neighborhood covers several tens of pixels and could possibly be extended to the whole image, see e.g., [32]; we drop the inclusion $\in \mathcal{N}_{i}$ in the following sums.
}

as to include in Eq.(7) only pixels corresponding to the same covariance $\boldsymbol{\Sigma}_{i}$. Designing methods to adaptively compute weights that preserve at best the resolution has been the subject of numerous works, starting with Lee's sigma filter [33] and oriented windows [34] up to more recent patchbased methods, see the review [35]. In the following, we chose to compute the weights using the NL-SAR algorithm [25] since it is very effective at preserving fine structures, and its parameters are tuned in an unsupervised way to adapt to the number of channels $D$, the sensor, the resolution and the image content. The derivation of our method is however general and independent from the choice of a specific algorithm for computing the weights $\omega_{i, j}$.

We define first the weighted maximum likelihood estimator $\widehat{\boldsymbol{\Sigma}}_{i}^{(\mathrm{WML})}$ as the covariance matrix $\boldsymbol{\Sigma}_{i}$ that minimizes $\mathcal{D}_{i}$.

Proposition 1. The weighted maximum likelihood estimator is given by the following weighted average:

$$
\widehat{\boldsymbol{\Sigma}}_{i}^{(\mathrm{WML})}=\frac{1}{\tau_{i}} \sum_{j} \omega_{i, j} \boldsymbol{g}_{j} \boldsymbol{g}_{j}^{\dagger} \quad \text { with } \quad \tau_{i}=\sum_{j} \omega_{i, j} .
$$

Proof. The definition of $\mathcal{D}_{i}$ in Eq.(7) leads to

$$
\begin{aligned}
\widehat{\boldsymbol{\Sigma}}_{i}^{(\mathrm{WML})} & =\underset{\boldsymbol{\Sigma}_{i}}{\operatorname{argmin}}-\sum_{j} \omega_{i, j} \log \mathrm{p}\left(\boldsymbol{g}_{j} \mid \boldsymbol{\Sigma}_{i}\right) \\
& =\underset{\boldsymbol{\Sigma}_{i}}{\operatorname{argmin}} \sum_{j} \omega_{i, j}\left[\log \operatorname{det}\left(\boldsymbol{\Sigma}_{i}\right)+\boldsymbol{g}_{j}^{\dagger} \boldsymbol{\Sigma}_{i}^{-1} \boldsymbol{g}_{j}\right] \\
& =\underset{\boldsymbol{\Sigma}_{i}}{\operatorname{argmin}} \tau_{i} \log \operatorname{det}\left(\boldsymbol{\Sigma}_{i}\right)+\sum_{j} \omega_{i, j} \operatorname{tr}\left[\boldsymbol{\Sigma}_{i}^{-1} \boldsymbol{g}_{j} \boldsymbol{g}_{j}^{\dagger}\right] \\
& =\underset{\boldsymbol{\Sigma}_{i}}{\operatorname{argmin}} \tau_{i} \log \operatorname{det}\left(\boldsymbol{\Sigma}_{i}\right)+\operatorname{tr}\left[\boldsymbol{\Sigma}_{i}^{-1}\left(\sum_{j} \omega_{i, j} \boldsymbol{g}_{j} \boldsymbol{g}_{j}^{\dagger}\right)\right] .
\end{aligned}
$$

The gradient of the objective function with respect to $\boldsymbol{\Sigma}_{i}$ is:

$$
\tau_{i} \boldsymbol{\Sigma}_{i}^{-\dagger}-\boldsymbol{\Sigma}_{i}^{-\dagger}\left(\sum_{j} \omega_{i, j} \boldsymbol{g}_{j} \boldsymbol{g}_{j}^{\dagger}\right) \boldsymbol{\Sigma}_{i}^{-\dagger} .
$$

After multiplying from the left and right by $\Sigma_{i}^{\dagger}$, the first order optimality condition (null gradient) leads to the desired result.

The expression of the data term $\mathcal{D}_{i}$ can be significantly simplified into a single term thanks to the following proposition:

Proposition 2. The weighted log-likelihood data term can be written in terms of the weighted maximum likelihood estimate:

$$
\mathcal{D}_{i}=\tau_{i}\left(\log \operatorname{det}\left(\boldsymbol{\Sigma}_{i}\right)+\operatorname{tr}\left[\boldsymbol{\Sigma}_{i}^{-1} \widehat{\boldsymbol{\Sigma}}_{i}^{(\mathrm{WML})}\right]\right) .
$$

Proof. The weighted log-likelihood is defined in Eq.(7) by:

$$
\begin{aligned}
\mathcal{D}_{i} & =-\sum_{j} \omega_{i, j} \log \mathrm{p}\left(\boldsymbol{g}_{j} \mid \boldsymbol{\Sigma}_{i}\right) \\
& =\sum_{j} \omega_{i, j}\left[\log \operatorname{det}\left(\boldsymbol{\Sigma}_{i}\right)+\boldsymbol{g}_{j}^{\dagger} \boldsymbol{\Sigma}_{i}^{-1} \boldsymbol{g}_{j}\right] \\
& =\tau_{i} \log \operatorname{det}\left(\boldsymbol{\Sigma}_{i}\right)+\operatorname{tr}\left[\boldsymbol{\Sigma}_{i}^{-1}\left(\sum_{j} \omega_{i, j} \boldsymbol{g}_{j} \boldsymbol{g}_{j}^{\dagger}\right)\right] \\
& =\tau_{i} \log \operatorname{det}\left(\boldsymbol{\Sigma}_{i}\right)+\tau_{i} \operatorname{tr}\left[\boldsymbol{\Sigma}_{i}^{-1} \widehat{\boldsymbol{\Sigma}}_{i}^{(\mathrm{WML})}\right]
\end{aligned}
$$


were the irrelevant additive constant term has been dropped, since $\mathcal{D}_{i}$ will be involved in minimization problems.

Proposition 2 has important practical consequences. While the original definition of the weighted log-likelihood data term $\mathcal{D}_{i}$ involved the sum of many terms (typically, several hundreds in the context of non-local methods) for a single pixel $i$, introduction of the weighted maximum likelihood estimate drastically simplifies the expression of $\mathcal{D}_{i}$ into a single term. This paves the way to a maximum a posteriori estimation based on data terms $\mathcal{D}_{i}$.

\section{B. Prior term}

In urban areas and at meter resolutions, the height is typically constant from one pixel to a neighboring pixel, or varies strongly when the two pixels belong to two different structures, e.g., ground and roof. We therefore select a prior term that favors piecewise constant images: the total variation defined by

$$
\sum_{(i, j)}\left|h_{i}-h_{j}\right|
$$

where $(i, j)$ indicates a pair of neighboring pixels.

Note that in other contexts (coarser resolutions, smooth surfaces), other convex pairwise regularization terms may be considered within the framework of our method.

\section{A posteriori energy $\mathcal{E}$}

The a posteriori energy for a height map $\boldsymbol{h}$, i.e., a vector of heights for all pixels, includes both the data term introduced in paragraph II-A, and the regularization proposed in paragraph II-B. As the data term is separable in terms of heights $h_{i}$, the a posteriori energy reads as:

$$
\mathcal{E}(\boldsymbol{h})=\sum_{i} \mathcal{D}_{i}\left(h_{i}\right)+\beta \sum_{(i, j)}\left|h_{i}-h_{j}\right|,
$$

where $\beta$ is a hyper-parameter that balances the relative importance of the fidelity to the observations (enforced by terms $\mathcal{D}_{i}$ ) and the smoothness of the height map $\boldsymbol{h}$ (enforced by the a priori). Beyond this global tuning through parameter $\beta$, it is necessary to account for the variable number of neighbors included in the weighted log-likelihood. Weights $\omega_{i, j}$ indeed vary from one pixel to another. In homogeneous regions, many similar neighbors are identified, thus the weighted maximum likelihood estimate $\widehat{\boldsymbol{\Sigma}}_{i}^{\text {(WML) }}$ is reliable. In contrast, in an isolated structure, very few similar neighbors are identified and most weights $\omega_{i, j}$ are (close to) zero, leading to a very noisy estimate $\widehat{\boldsymbol{\Sigma}}_{i}^{\text {(WML) }}$. To account for this disparity between estimates, we follow the idea of [27] and set the sum of weights $\tau_{i}$ at pixel $i$ (see Eq.(8)) to be inversely proportional to the standard deviation of the estimator:

$$
\tau_{i}=\sqrt{\hat{L}_{i}}
$$

with $\hat{L}_{i}$ the equivalent number of looks corresponding to the weighted neighborhood defined by the weights $\omega_{i, j}$ [25]:

$$
\hat{L}_{i}=\frac{\left(\sum_{j} \omega_{i, j}\right)^{2}}{\sum_{j} \omega_{i, j}^{2}} .
$$

\section{MAP estimation of the height distribution}

The height map $\boldsymbol{h}$ can be estimated in the MAP sense by solving the minimization problem:

$$
\hat{\boldsymbol{h}}^{\text {(MAP) }}=\underset{\boldsymbol{h}}{\operatorname{argmin}} \mathcal{E}(\boldsymbol{h}),
$$

whose expression can be recast as follow.

Proposition 3. Let $\hat{r}_{a}, \hat{\gamma}_{a, b}$ and $\hat{\psi}_{a, b}$ be the estimated reflectivities, coherences and phases extracted from $\widehat{\mathbf{\Sigma}}_{i}^{(\mathrm{WML})}$ using Eq.(2) and (3). Consider reflectivity values and coherences to be fixed, i.e., $\hat{r}_{a}=r_{a}$ and $\hat{\gamma}_{a, b}=\gamma_{a, b}$ for all channels $a$ and $b$, and optimize only with respect to the height (no joint optimization). The energy minimization problem (14) becomes:

$$
\begin{aligned}
& \hat{\boldsymbol{h}}^{(\mathrm{MAP})}=\underset{\boldsymbol{h}}{\operatorname{argmin}} \sum_{i} \sqrt{\hat{L}_{i}} \cdot \operatorname{tr}\left[\boldsymbol{\Gamma}_{i}^{-1}\left(h_{i}\right) \cdot \widehat{\boldsymbol{\Gamma}}_{i}^{(\mathrm{WML})}\right] \\
& +\beta \sum_{(i, j)}\left|h_{i}-h_{j}\right|, \\
& \text { with }\left[\boldsymbol{\Gamma}_{i}\left(h_{i}\right)\right]_{a, b}=\hat{\gamma}_{a, b} \cdot \exp \left(j \cdot f_{a, b}\left(h_{i}\right)\right) \\
& \text { and }\left[\widehat{\boldsymbol{\Gamma}}_{i}^{(\mathrm{WML})}\right]_{a, b}=\hat{\gamma}_{a, b} \cdot \exp \left(j \cdot \hat{\psi}_{a, b}\right) \text {, }
\end{aligned}
$$

where $f_{a, b}$ is defined in Eq.(4).

Proof. Since $\operatorname{det}\left(\boldsymbol{\Sigma}_{i}\right)$ does not depend on $h_{i}$, see [36], the $\log \operatorname{det}\left(\boldsymbol{\Sigma}_{i}\right)$ terms in Eq.(9) can be dropped in the data terms $\mathcal{D}_{i}$. The energy minimization problem becomes:

$$
\begin{gathered}
\hat{\boldsymbol{h}}^{(\mathrm{MAP})}=\underset{\boldsymbol{h}}{\operatorname{argmin}} \sum_{i} \sqrt{\hat{L}_{i}} \cdot \operatorname{tr}\left[\boldsymbol{\Sigma}_{i}^{-1}\left(h_{i}\right) \cdot \widehat{\boldsymbol{\Sigma}}_{i}^{(\mathrm{WML})}\right] \\
+\beta \sum_{(i, j)}\left|h_{i}-h_{j}\right|
\end{gathered}
$$

As $\hat{r}_{a}=r_{a}$, we have $\widehat{\boldsymbol{\Sigma}}_{i}^{(\text {WML })}=\boldsymbol{R}_{i}^{1 / 2} \widehat{\boldsymbol{\Gamma}}_{i}^{(\text {WML })} \boldsymbol{R}_{i}^{1 / 2}$ and $\boldsymbol{\Sigma}_{i}^{-1}\left(h_{i}\right)=\boldsymbol{R}_{i}^{-1 / 2} \boldsymbol{\Gamma}_{i}^{-1}\left(h_{i}\right) \boldsymbol{R}_{i}^{-1 / 2}$. Injecting these two equalities in the above equation, and using that $\gamma_{a, b}=\hat{\gamma}_{a, b}$ and $\psi_{a, b}=f_{a, b}\left(h_{i}\right)$ conclude the proof.

This minimization problem is highly non-convex because of the dependence on $\boldsymbol{h}$ in the data term through a phase term. Global minimization can still be performed since the data term is separable (a sum of independent terms over all pixels) and the regularization is a sum of convex pairwise terms (i.e., involving only pairs of pixels). We use the graph construct of Ishikawa [37] to map the original non-convex problem into a maximum-flow / minimum-cut problem. We discretize the range of height values into $H$ heights, then build a graph with $H$ layers, each layer containing a node for each pixel in the image. Each node is connected to nodes corresponding to the spatial neighbors within each layer, and to the corresponding nodes in the layer immediately above and below. Capacities of the edges are set according to values of the terms in the optimization problem (15). 
The size of the graph is thus proportional to the number of pixels times the number of heights. Memory constraints therefore limit the method to regions of size below a million pixels. Larger regions can be processed either by considering sliding windows, as in [38], or by using multilabel partition moves [39]. If a different convex and pairwise regularization term was preferred (see paragraph II-B), a similar graph construct would still be possible but it would involve many supplementary arcs. The convex optimization approach described in [40] would then be preferable in terms of computational and memory costs. The choice of a non-convex regularization term (e.g., a truncated quadratic function of the height differences) would make the optimization much harder and only approximate solutions could be sought, at the risk of falling in a local minimum due to the multi-modal nature of the data term $\mathcal{D}_{i}$.

The height reconstruction algorithm, called PARISAR (PAtch-based estimation and Regularized Inversion for SAR interferometry), is summarized in the following box.

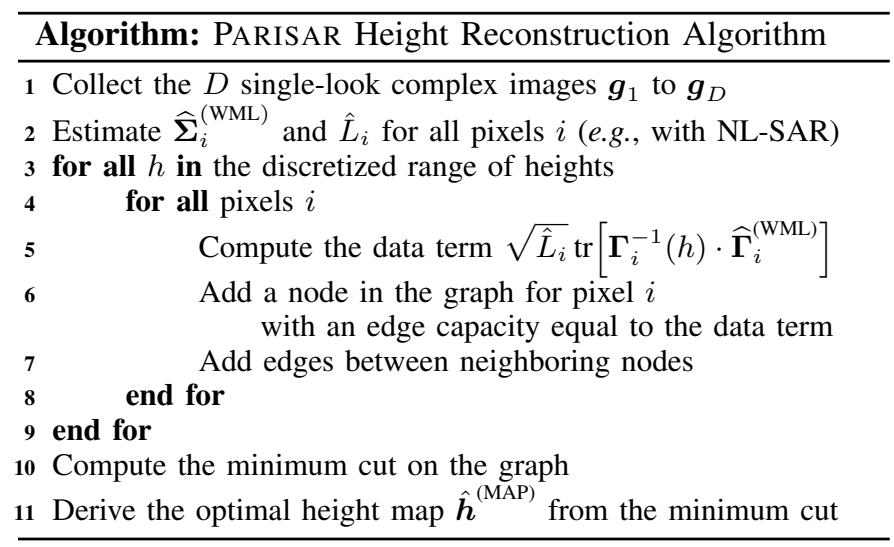

\section{VALIDATION ON NUMERICAL SIMULATIONS}

The quantitative and qualitative assessment of the method has been conducted on different test cases. First a quantitative validation is performed using three different simulated test cases: an urban-like scenario, a pattern of squares and a natural scenario, named Ghiglia. The first test case aims at validating the ability of the proposed approach to unwrap and regularize areas characterized by height discontinuities, to correctly handle very low coherence areas (for example shadow areas), and to retrieve small scale structures. The second simulation is designed to analyze the performance of the method for a wide range of configurations (i.e., different coherence and building height values). The last simulation, Ghiglia, is a classical numerical simulation used to assess the behavior of algorithms in a case close to natural height maps.

The datasets are made of three complex images corresponding to different baselines. The adopted system parameters are summarized in Table I. In particular, the table contains the $\alpha_{a, b}$ parameters of Eq.(4), the corresponding ambiguity height and the size of the considered scene. In the following, parameter $\beta$ has been manually set. The adopted values are reported for the different datasets.

In order to compare the results provided by the proposed algorithm PARISAR, different multi-channel methods have been considered: MLNL (Maximum Likelihood with Non Local), MAPNL (Maximum a Posteriori with Non Local) and MCPU (Multi-Channel Phase Unwrapping). The first one is a weighted maximum likelihood estimator that exploits the weighted $\log$-likelihood terms $\mathcal{D}_{i}$, without any regularization; the second one is a Maximum a Posteriori that implements the estimator of Eq.(15) with a sub-optimal minimization procedure based on Iterated Conditional Modes (ICM)); finally the MCPU proposed in [23] implements a Maximum a Posteriori estimator based on the statistical independence between interferograms and on the use of a graph-cut-based optimal minimization procedure.

The processing is performed by a high-level code written in Matlab language and based on $\mathrm{C} / \mathrm{C}++$ code for NL-SAR filtering and minimum cut computations, on an Intel Core i7 workstation with Linux Debian as operative system.

\section{A. Urban-like profile}

The complex data have been simulated starting from the height profile and the coherence map, reported in Figures 1(f) and 1(a). The starting height profile is a typical scenario used to assess the capability of multi-channel algorithms, made of structures of different heights, characterized by height discontinuities. The characteristics of the profile and of the scene are reported in Table I. The height of the buildings are such that the related phases are ambiguous even for the smallest considered baseline, making the unwrapping of the profile a difficult task, even in the absence of noise. Concerning the coherence of the scene, three areas have been considered: ground and buildings area, top left building, shadowing areas (see Figure 1(a)). For the generation of the data, the following values were adopted for the different combination of the three images: $\gamma=\{0.7,0.65,0.6\}$ for the ground and buildings area, $\gamma=\{0.45,0.35,25\}$ for the top left building, $\gamma=\{0.1,0.1,0.1\}$ for the shadowing areas. Note that for the considered profile and the adopted coherence the Itoh condition is not satisfied, thus a singlechannel unwrapping algorithm can not be adopted.

The generated $D=3$ interferograms are shown in Figures 1(c), 1(d), 1(e). The mean estimated coherence map, using a simple box-car filter, is shown in Figure 1(b).

PARISAR and the other previously reported multi-channel algorithms have been tested on the dataset. From the visual inspection of the results, the good performances of the proposed algorithm are evident. While all the other considered techniques either fail in estimating the height of some buildings (MCPU), or fail in removing the noise (MLNL) or fail in retrieving the details of the image, such as borders, the small structures or shadowing areas (MAPNL), PARISAR is able to correctly solve all the previously reported issues. The image is well regularized, all the structures, with the correct heights, are retrieved. Shadow areas are well reconstructed and the small structure is not flattened or confused with the surrounding ground area. A strong reduction of the variance of height estimation while preserving edges (no blurring phenomenon) is achieved.

The visual analysis is confirmed by the quantitative analysis based on the evaluation of the Normalized Reconstruction 
TABLE I

Numerical SimUlations PaRAMETERS (SIZES GIVEN IN PIXELS).

\begin{tabular}{|c|c|c|c|}
\hline Dataset & $\alpha_{a, b}$ & $h_{a m b}$ & size \\
\hline Urban & {$\left[\begin{array}{lll}-0.55 & -1 & -0.45\end{array}\right]$} & {$\left[\begin{array}{lll}5.7 & 3.1 & 6.9\end{array}\right] \mathrm{m}$} & $70 \times 70$ \\
\hline Squares & {$\left[\begin{array}{lll}-0.55 & -1 & -0.45\end{array}\right]$} & {$\left[\begin{array}{lll}5.7 & 3.1 & 6.9\end{array}\right] \mathrm{m}$} & $240 \times 240$ \\
\hline Ghiglia & {$\left[\begin{array}{lll}-0.55 & -1.2 & -0.65\end{array}\right]$} & {$\left[\begin{array}{llll}5.7 & 2.6 & 4.8\end{array}\right] \mathrm{m}$} & $458 \times 157$ \\
\hline
\end{tabular}

TABLE II

NormaLIZED RECONSTRUCTION SQUARE ERROR

\begin{tabular}{lccccc}
\hline Dataset & MLNL & MAPNL & MCPU & PUMA & PARISAR \\
\hline Urban & 1.16 & 0.32 & 0.29 & - & 0.03 \\
Squares & 3.88 & 1.07 & 1.12 & - & 0.52 \\
Ghiglia & 0.29 & 0.13 & - & 0.01 & 0.001 \\
\hline
\end{tabular}

Square Error, defined as the quadratic norm of the difference between the true height values and the estimated ones, normalized by the the true ones (see [22]) of Table II and on the Root Mean Square Error, reported in Table III. For both parameters, PARISAR outperforms the other algorithms.

\section{B. Pattern of squares}

This second test aims at analyzing the proposed method for different configurations, in terms of height and coherence values. The starting true profile is made of structures of different heights (Figure 2(f)), while the coherence spans different values from 0 to 1 (Figure 2(a)). By combining the true profile and the coherence map with the different available baselines (system parameters are reported in Table I), three complex images are generated. The effect of this combination is an interferogram characterized by different behaviors: the top left part contains low height structures with weak noise (high coherence value), while the bottom right corner is characterized by high heights with strong noise. The other two quadrants contain low height structures under strong noise and high height structures under a weak noise.

The dataset is used to test all previously mentioned algorithms. The results are shown in the second row of Figure 2. MLNL and MCPU provide unsatisfying results: the former provides a noisy solution, while the latter over-regularizes the solution creating artifacts. The best results are achieved in case of MAPNL and PARISAR. Exploiting the non-local estimation both techniques are able to provide effective results in almost all the areas. PARISAR outperforms MAPNL, in terms of noise regularization and correct height retrieval. This is evident from both visual inspection and quantitative analysis, reported in Tables II and III (see the corresponding lines for the Square dataset). As expected, the errors that appear in PARISAR reconstruction are mainly in the bottom line, where the interferometric noise is strong.

\section{Ghiglia profile}

In order to assess the performances of the algorithm on a natural scenario, a realistic profile generated on the basis
TABLE III

RoOT MEAN SQUARE ERROR

\begin{tabular}{lccccc}
\hline Dataset & MLNL & MAPNL & MCPU & PUMA & PARISAR \\
\hline Urban & 3.11 & 1.63 & 1.56 & - & 0.52 \\
Squares & 5.3 & 2.78 & 2.84 & - & 1.94 \\
Ghiglia & 17.41 & 11.47 & - & 3.96 & 1.22 \\
\hline
\end{tabular}

of a real digital elevation model of mountainous terrain around Isolation Peak, Colorado, is considered [41]. In the following we will refer to it as Ghiglia profile. The system parameters are reported in Table I. Three different coherence values, $\{0.7,0.65,0.6\}$, are adopted, for the three considered combinations of images. Three interferograms are generated. The true profile, the data and the results are reported in Figure 3 . The considered profile is not ambiguous: there are no height discontinuities. In this case, the unwrapping task difficulty comes from the fringes that tend to overlap, creating a sort of aliasing. Since the profile is not ambiguous, a single-channel phase unwrapping can be used to unwrap the profile. The PUMA algorithm proposed in [42] is considered.

The results obtained using MAPNL, PUMA and PARISAR are shown in Figures 3(f), 3(g) and 3(h), respectively. The first and the last are tested using the whole dataset, PUMA is tested using only the smallest baseline interferogram. It is evident that, even if not ambiguous, a single-channel algorithm as PUMA is penalized by using a single interferogram and therefore fails to correctly retrieve the height, due to the aliasing of fringes. This problem is solved by PARISAR by using all the available channels. Note that exploiting the whole dataset may not be sufficient for correctly retrieving the profile: the difference between results obtained by MAPCorrNL and PARISAR show that the regularization role is important. The quantitative analysis reported in in Tables II and III (see the corresponding lines for the Ghiglia dataset) confirms the visual inspection.

\section{APPLICATION TO SATELlite SAR IMAGES}

To qualitatively evaluate algorithm PARISAR on real satellite SAR images, we considered two datasets: an urban test site (Napoli) and a natural test site (Serre-Ponçon). The two scenes have been acquired using two different sensors, COSMOSkyMed and ERS, to test the capabilities of the method to work with different radar frequencies (X-band and C-band) and sensors. The systems parameters, previously defined in Eq.(4), are summarized in table IV. For both datasets, the hypothesis of stationary observed scene is considered. This hypothesis is met when the temporal baseline span is limited. For the considered data sets, the maximum temporal baseline span is of 4 months for Napoli test case and 5 months for Serre-Ponçon one, which are compatible with the stationary hypothesis.

A pre-processing procedure is mandatory for all multi-channel based algorithms: it is needed to correctly combine the different available images. The pre-processing consists of two steps: the first one aims at removing possible phase artifacts (due for 


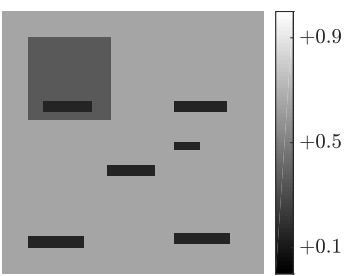

(a)

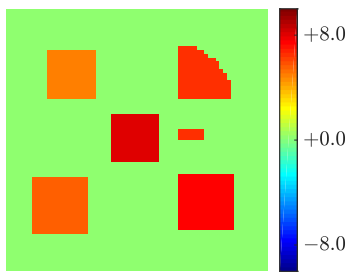

(f)

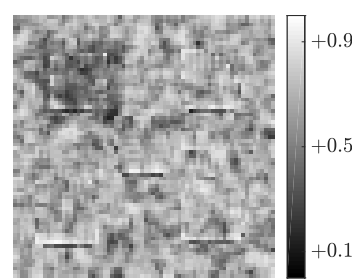

(b)

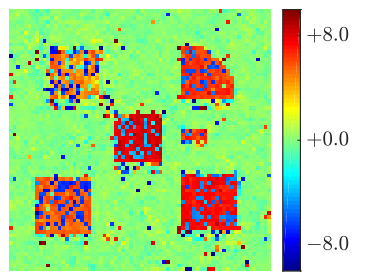

(g)

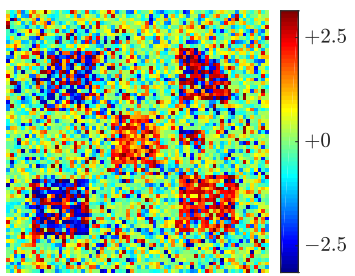

(c)

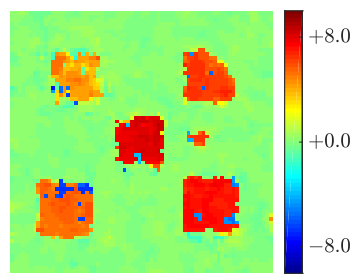

(h)



(d)

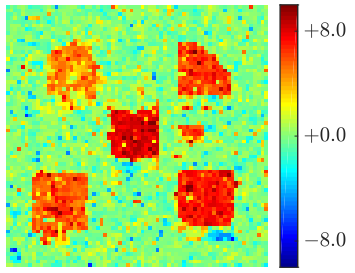

(i)

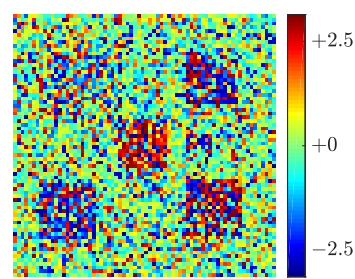

(e)

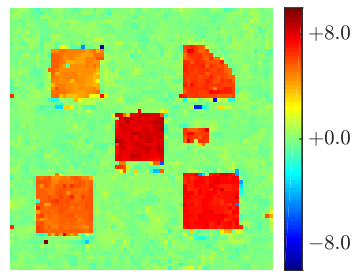

(j)

Fig. 1. (a) Starting coherence map, (b) empirical coherence map, (c) first interferogram, (d) second interferogram, (e) third interferogram, (f) original height profile, (g) estimated solution using MLNL approach, (h) estimated solution using MAPNL approach, (i) estimated solution using MCPU approach, (j) estimated solution using the proposed PARISAR approach, for $\beta=0.25$.



(a)

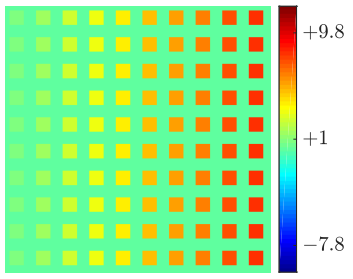

(f)



(b)

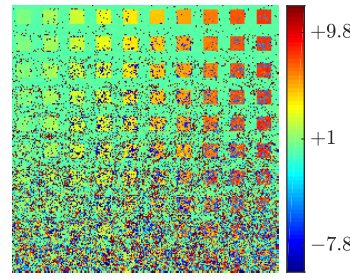

(g)

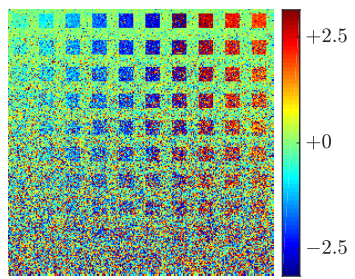

(c)

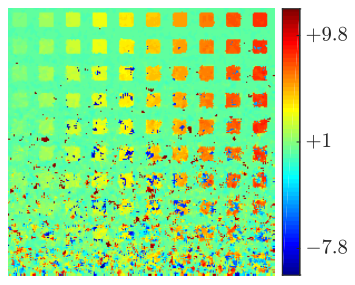

(h)

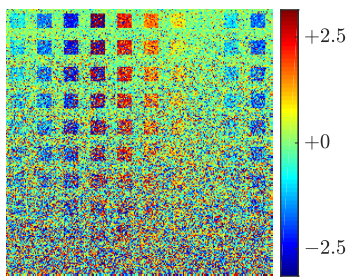

(d)

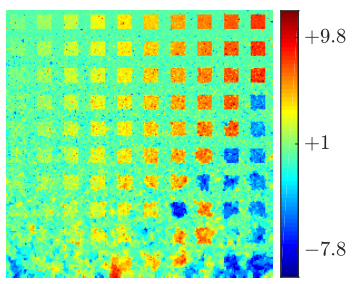

(i)

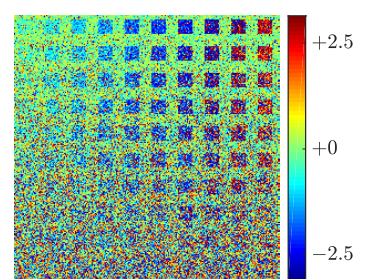

(e)

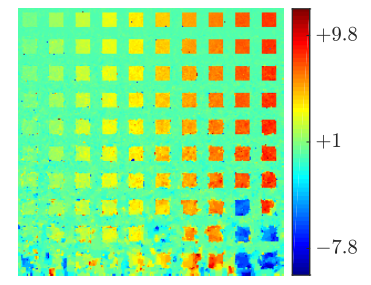

(j)

Fig. 2. (a) Starting coherence map, (b) empirical coherence map, (c) first interferogram, (d) second interferogram, (e) third interferogram, (f) original height profile, (g) estimated solution using MLNL approach, (h) estimated solution using MAPNL approach, (i) estimated solution using MCPU approach, (j) estimated solution using the proposed PARISAR approach, for $\beta=0.05$.

example to the atmosphere), while the second one consists in the calibration of the phases. Concerning the first step, different techniques can be applied according to the extension and to the topography of the observed scene. The algorithm proposed in [43] has been adopted for Napoli test case, while the algorithm proposed in [44] has been considered for SerrePonçon test case. Concerning the second step, commonly a relative phase calibration is applied based on the identification of high coherence areas or permanent scatters. After the reconstruction a constant offset is applied to the final image (e.g. commonly the value of the offset is such that the ground is set to zero meters).

\section{A. Urban area: Napoli test case}

The first dataset is composed of three $250 \times 250$ pixels COSMO-SkyMed Stripmap images acquired close to Naples
TABLE IV

INTERFEROMETRIC CONFIGURATIONS OF THE SATELLITE IMAGES

\begin{tabular}{|c|c|c|c|c|c|}
\hline Dataset & Sensor & $\rho_{0}$ & $\lambda$ & $\theta$ & $B_{\perp}$ \\
\hline Naples & CSK & 755.190 & 0.03 & 0.62 & {$\left[\begin{array}{llll}0 & 517 & 251\end{array}\right] \mathrm{m}$} \\
\hline Serre-P. & ERS2 & 825.669 & 0.05 & 0.40 & {$\left[\begin{array}{llll}0 & 36 & 96\end{array}\right] \mathrm{m}$} \\
\hline
\end{tabular}

train station, in Italy. One of the three available interferograms is shown in the first row of Figure 4 together with the mean amplitude (in log scale) and the mean coherence map. The scene is very complex: different structures, with different heights, shapes and reflectivities are present. The phase unwrapping results are shown in the second row. Independent estimation of the height at each pixel leads to a very noisy result (i.e., strong variance of the heights). This is evident from 


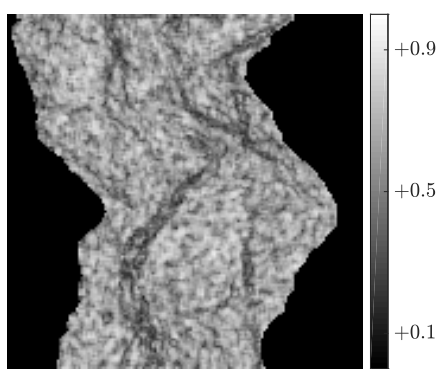

(a)

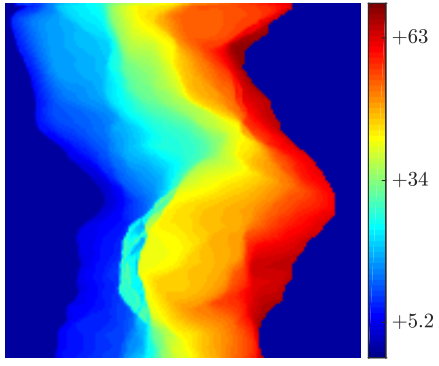

(e)



(b)

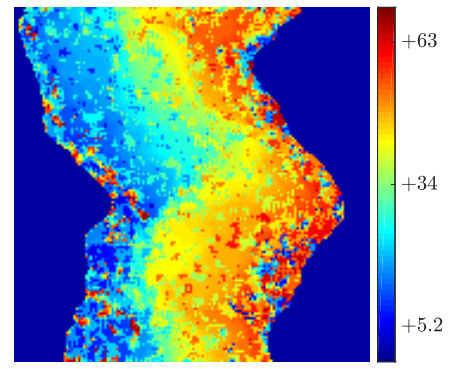

(f)

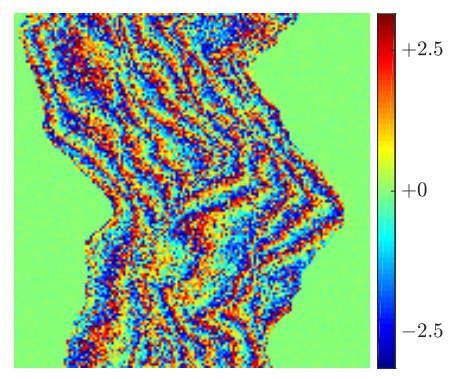

(c)

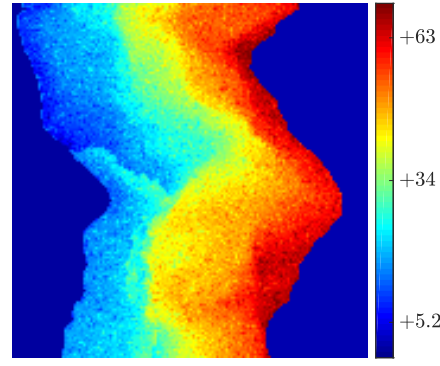

(g)

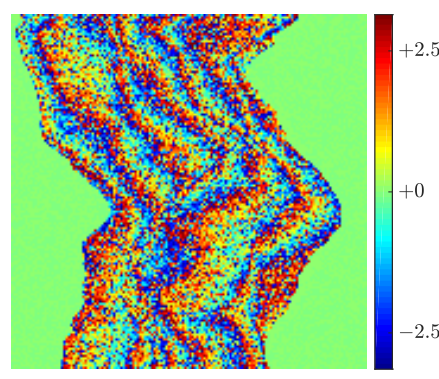

(d)

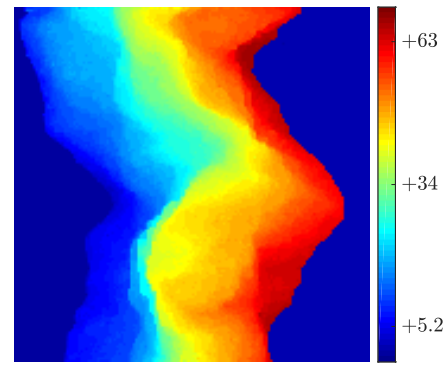

(h)

Fig. 3. (a) Empirical coherence map, (b) first interferogram, (c) second interferogram, (d) third interferogram, (e) original height profile, (f) estimated solution using MAPNL approach, (g) estimated solution using PUMA approach, (h) estimated solution using the proposed PARISAR approach, for $\beta=0.1$.

the results of MLNL of Figure 4(e). Considering MAPNL and PARISAR, the regularization reduces these fluctuations without noticeable resolution loss. This reduction is more evident in case of PARISAR (Figure 4(g)): the building structures are retrieved, both in terms of shapes and heights. It is interesting to note the capability of PARISAR in retrieving the low-height circular structures on the left of the scene, while strongly reducing noise: these structures are almost invisible both in the interferogram and in the coherence image (very noisy area). A quantitative validation of the result for Napoli test case can be performed using Google Earth height data. The height of the large building on the left of the scene, provided by Google Earth, is of about $23 \mathrm{~m}$ (highest area) and $18 \mathrm{~m}$, while the height of the ground is of $8 \mathrm{~m}$. All Google Earth data refer to the sea level. The relative height of the building is compatible with the reconstruction of PARISAR. The height of the circular building on the right of the scene, provided by Google Earth, is of about $14 \mathrm{~m}$, while the height of the ground is of $7 \mathrm{~m}$, on the sea level. The relative height of the building is also compatible with the reconstruction of PARISAR. A qualitative evaluation of the reconstruction can be performed based on the optical images (2D and 3D) of the considered scene provided by Google (Figures 4(d) and 4(h)), taken at the same time period: from the radar-optical comparison it appears that the structures are correctly retrieved, both in terms of shapes and of relative building heights.

\section{B. Mountainous area: Serre-Ponçon test case}

The last $250 \times 250$ pixel dataset corresponds to a mountainous area acquired by ERS sensor over Serre-Ponçon (France). One of the three available interferograms is shown in the first row of Figure 5 together with the mean amplitude (in log scale) and the mean coherence map. This area is challenging due to the presence of very low coherence areas and not regular phase fringes. Phase unwrapping results are shown in the second row. Both MLNL and MAPNL fail at correctly unwrapping the profile. The latter provides a more reliable result although there are several areas that are not correctly unwrapped. Using PARISAR, it is possible to largely improve the results. Wrapping problems are solved and noise is better suppressed.

\section{CONClusion}

A new methodology to improve multi-baseline phase unwrapping has been proposed. Starting from the complete statistical distribution of the interferometric data, the joint exploitation of patch-based approaches and TV regularization for elevation estimation has been discussed. The developed algorithm, named PARISAR, implements a maximum a posteriori estimator with a properly modified likelihood term, by means of a two steps strategy: the first step consists of estimating a covariance matrix at each pixel from the multichannel images available using a non-local filtering method like NL-SAR; the second step introduces a TV penalty for edge-preserving regularization. PARISAR has been tested on several datasets and compared to other multi-channel algorithms. The quantitative and qualitative analysis has been carried out on three different simulated datasets, in order to validate the effectiveness of the approach in different configurations (various image structures and coherences). A qualitative evaluation has been performed on two satellite image datasets from two different sensors working at different radar frequencies, displaying different spatial resolutions, on an urban and a mountainous area. The results in both cases are promising. PARISAR provides sensible elevation profiles, seemingly outperforming other methods. Structural details are 


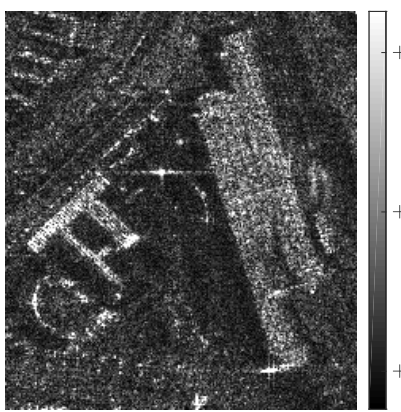

(a)

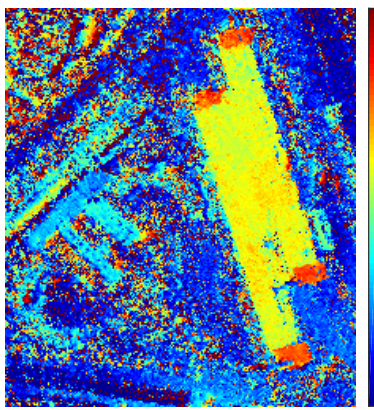

(e)

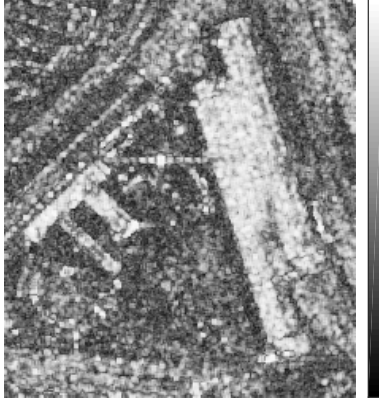

(b)

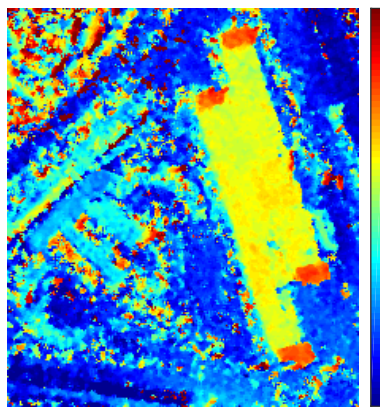

(f)
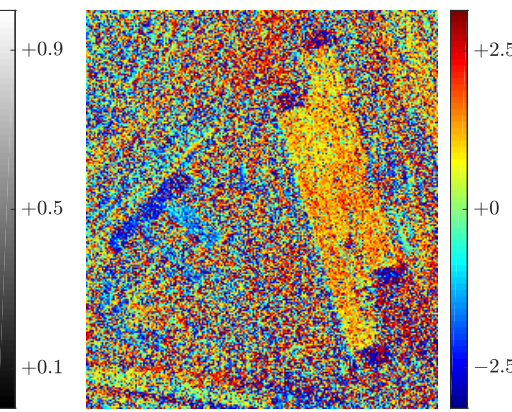

(c)

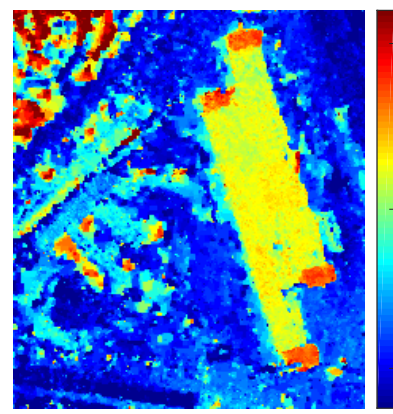

(g)

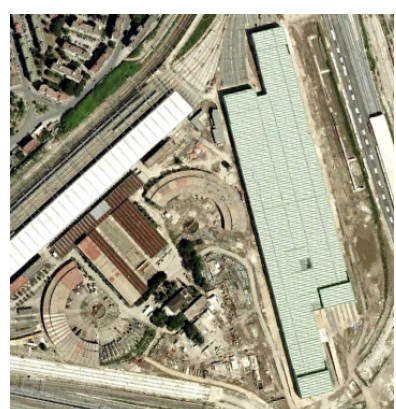

(d)

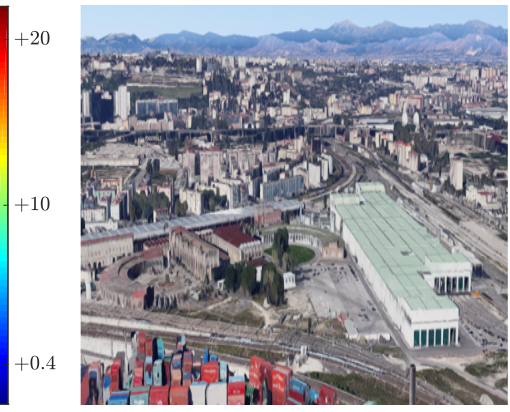

(h)

Fig. 4. (a) Mean amplitude image, (b) empirical coherence map, (c) one of the available interferograms, (d) optical image of the considered scene provided by Google, (e) estimated solution using MLNL approach, (f) estimated solution using MAPNL approach, (g) estimated solution using the proposed method PARISAR for $\beta=0.05$, (h) 3D optical image of the considered scene provided by Google Earth.

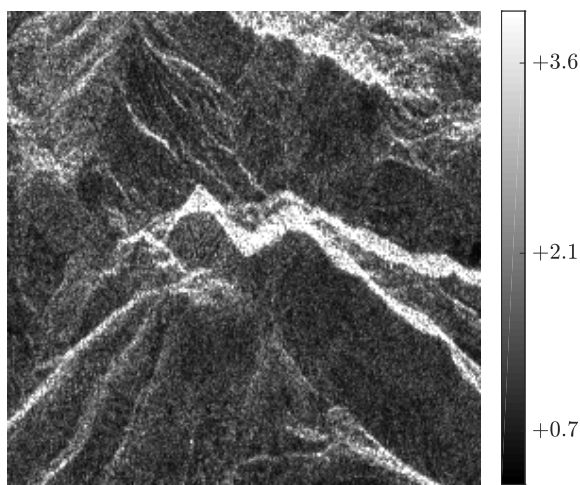

(a)

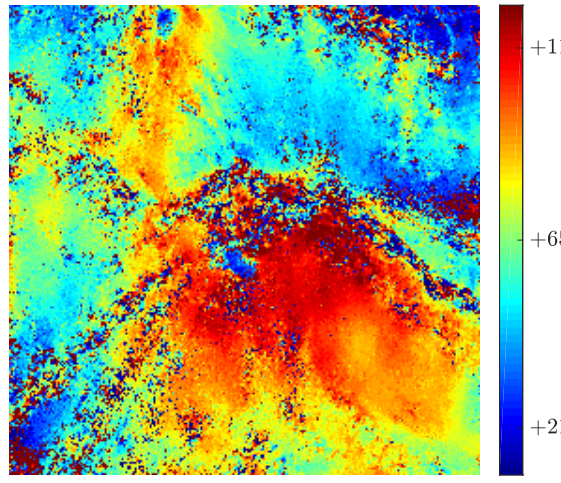

(d)

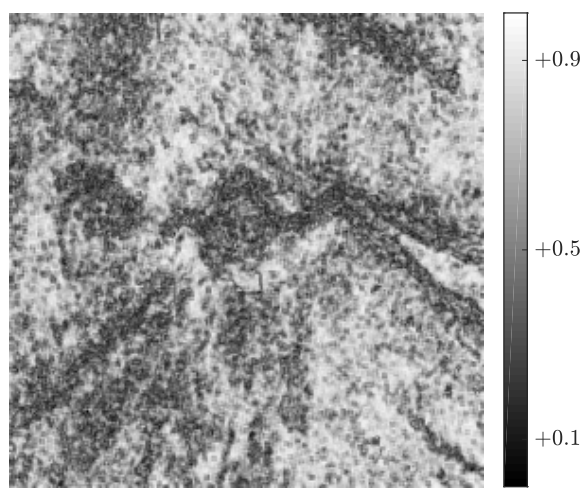

(b)

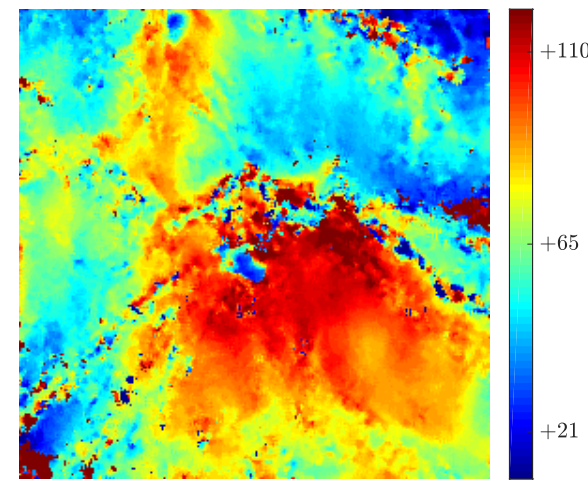

(e)

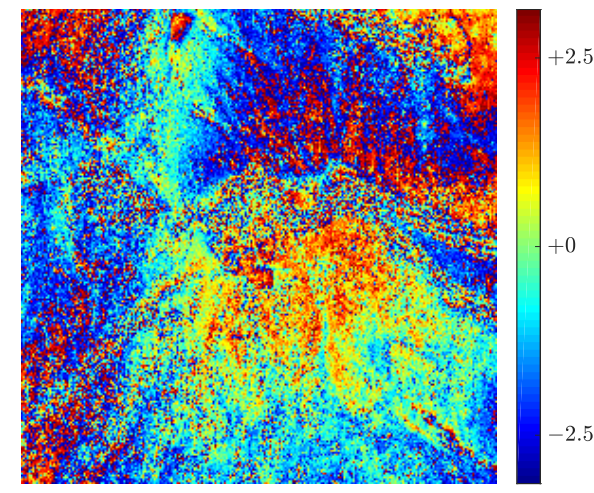

(c)

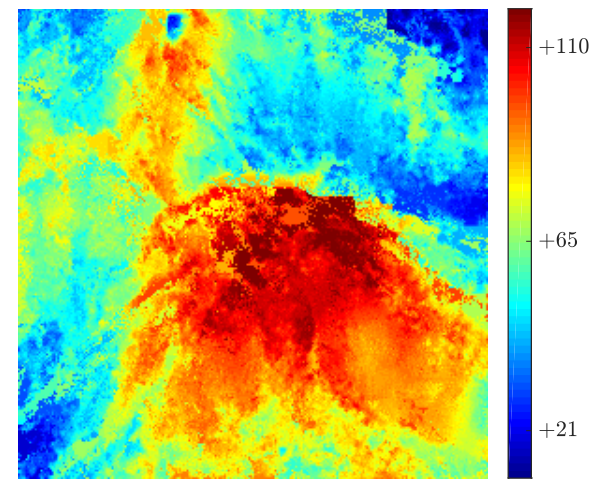

(f)

Fig. 5. (a) One of the available amplitude, (b) empirical coherence map, (c) one of the available interferogram, (d) estimated solution using MLNL approach, (e) estimated solution using MAPNL approach, (f) estimated solution using the proposed method PARISAR, for $\beta=0.5$. 
preserved while most of the noise is suppressed. All the considered datasets were composed of only three images, to show the potentiality of the technique in working with a very limited number of images (avoiding problems with large temporal baselines such as de-correlations, deformations, etc.). Clearly a larger number of images, if available, can be used by PARISAR. If the pre-processing of the images is correctly performed the reconstruction would improve, since the log-likelihood energy would benefit of additional data. At the present stage, the method is able to handle shadows but it does not accounts for the layover phenomenon. Only tomographic approaches are able to provide a solution in the case where different echoes, from structures at different heights (i.e. roof, facade and ground), are integrated within the same resolution cell. Interferometric approaches could be used in such layover areas only in the case where one of the contributions is dominant compared to the others. This sometimes happens with the facades of the buildings that are characterized by stronger reflections compared to the roof and the ground. In this situation, the known layover ramp appears in the interferograms (see [45], [46]) and the proposed PARISAR algorithm would correctly manage and reconstruct the ramp. On the contrary, if there is no dominant contribution, such distortions can be addressed only using a tomographic approach. The investigation of a tomographicbased approach within the proposed framework is the subject of future research.

\section{ACKNOWLEDGMENTS}

This work has been partly supported by the Italian Space Agency (ASI) within the project ID2246 Development of Imaging and Monitoring Methodologies based on the use of COSMO-Skymed SAR data - Contract n. I/065/09/0. This work has been supported by ANR (the French National Research Agency) and DGA (Direction générale de l'Armement) under ALYS Project ANR-15-ASTR-0002.

\section{REFERENCES}

[1] G. Fornaro and V. Pascazio, "SAR Interferometry and Tomography: Theory and Applications," Academic Press Library in Signal Processing, Elsevier, 2013.

[2] R. Bamler and P. Hartl, "Synthetic Aperture radar interferometry," Inverse Problem, vol. 14, pp. R1-R54, August 1998.

[3] R. Chen, W. Yu, R. Wang, G. Liu, and Y. Shao, "Integrated Denoising and Unwrapping of InSAR Phase Based on Markov Random Fields," IEEE Transactions on Geoscience and Remote Sensing, vol. 51, no. 8, pp. 4473-4485, Aug 2013.

[4] B. Osmanoğlu, F. Sunar, S. Wdowinski, and E. Cabral-Cano, "Time series analysis of InSAR data: Methods and trends," ISPRS Journal of Photogrammetry and Remote Sensing, vol. 115, pp. 90 - 102, 2016.

[5] K. Itoh, "Analysis of the phase unwrapping problem," Appl. Opt., vol. 21, no. 14, 1982.

[6] V. Pascazio and G. Schirinzi, "Multifrequency InSAR height reconstruction through maximum likelihood estimation of local planes parameters," IEEE Transactions on Image Processing, vol. 11, no. 12, pp. 1478-1489, 2002.

[7] G. Ferraiuolo, F. Meglio, V. Pascazio, and G. Schirinzi, "DEM Reconstruction Accuracy in Multichannel SAR Interferometry," IEEE Transactions on Geoscience and Remote Sensing, vol. 47, no. 1, pp. 191 $-201,2009$.

[8] F. Li and R. Goldstein, "Studies of multibaseline spaceborne interferometric synthetic aperture radars," IEEE Transactions on Geoscience and Remote Sensing, vol. 28, no. 1, pp. 88-97, 1990.
[9] A. Ferretti, C. Prati, and F. Rocca, "Multibaseline phase unwrapping for InSAR topography estimation," Nuovo cimento della Societa italiana di fisica. C, Geophysics and space physics, vol. 24, no. 1, pp. 159-176, 2001.

[10] F. Gini and F. Lombardini, "Multibaseline cross-track SAR interferometry: a signal processing perspective," IEEE Aerospace and Electronic Systems Magazine, vol. 20, no. 8, pp. 71-93, Aug 2005.

[11] G. Fornaro, A. M. Guarnieri, A. Pauciullo, and F. De-Zan, "Maximum liklehood multi-baseline SAR interferometry," IEE Proceedings - Radar, Sonar and Navigation, vol. 153, no. 3, pp. 279-288, June 2006.

[12] P. Berardino, G. Fornaro, R. Lanari, and E. Sansosti, "A new algorithm for surface deformation monitoring based on small baseline differential sar interferograms," IEEE Transactions on Geoscience and Remote Sensing, vol. 40, no. 11, pp. 2375-2383, 2002.

[13] A. Ferretti, A. Fumagalli, F. Novali, C. Prati, F. Rocca, and A. Rucci, "A new algorithm for processing interferometric data-stacks: Squeesar," IEEE Transactions on Geoscience and Remote Sensing, vol. 49, no. 9, pp. 3460-3470, 2011.

[14] H. Liu, M. Xing, and Z. Bao, "A Cluster-Analysis-Based Noise-Robust Phase-Unwrapping Algorithm for Multibaseline Interferograms," IEEE Transactions on Geoscience and Remote Sensing, vol. 53, no. 1, pp. 494-504, 2015.

[15] H. Liu, M. Xing, and Z. Bao, "A Novel Mixed-Norm Multibaseline Phase-Unwrapping Algorithm Based on Linear Programming," IEEE Geoscience and Remote Sensing Letters, vol. 12, no. 5, pp. 1086-1090, 2015.

[16] D. Chirico and G. Schirinzi, "Multichannel interferometric SAR phase unwrapping using extended Kalman Smoother," International Journal of Microwave and Wireless Technologies, vol. 5, pp. 429-436, 62013.

[17] M. Schmitt and U. Stilla, "Maximum-likelihood estimation for multiaspect multi-baseline SAR interferometry of urban areas," ISPRS Journal of Photogrammetry and Remote Sensing, vol. 87, no. 0, pp. $68-77,2014$.

[18] A. Shabou, F. Baselice, and G. Ferraioli, "Urban digital elevation model reconstruction using very high resolution multichannel InSAR data," IEEE Transactions on Geoscience and Remote Sensing, vol. 50, no. 11, pp. 4748-4758, 2012.

[19] M. Eineder and N. Adam, "A maximum-likelihood estimator to simultaneously unwrap, geocode, and fuse SAR interferograms from different viewing geometries into one digital elevation model," IEEE Transactions on Geoscience and Remote Sensing, vol. 43, no. 1, pp. 24-36, 2005.

[20] G. Ferraiuolo, V. Pascazio, and G. Schirinzi, "Maximum a posteriori estimation of height profiles in InSAR imaging," IEEE Geoscience and Remote Sensing Letters, vol. 1, no. 2, pp. 66-70, 2004.

[21] G. Nico, G. Palubinskas, and M. Datcu, "Bayesian approaches to phase unwrapping: theoretical study," IEEE Transactions on Signal Processing, vol. 48, no. 9, pp. 2545-2556, 2000

[22] F. Baselice, G. Ferraioli, V. Pascazio, and G. Schirinzi, "Contextual information-based multichannel synthetic aperture radar interferometry: Addressing DEM reconstruction using contextual information," IEEE Signal Processing Magazine, vol. 31, no. 4, pp. 59-68, July 2014.

[23] G. Ferraioli, A. Shabou, F. Tupin, and V. Pascazio, "Multichannel phase unwrapping with graph cuts," IEEE Geoscience and Remote Sensing Letters, vol. 6, no. 3, pp. 562-566, 2009.

[24] G. Ferraiuolo and G. Poggi, "A Bayesian filtering technique for SAR interferometric phase fields," IEEE Transactions on image processing, vol. 13, no. 10, pp. 1368-1378, 2004.

[25] C.-A. Deledalle, L. Denis, F. Tupin, A. Reigber, and M. Jäger, "NLSAR: A unified nonlocal framework for resolution-preserving (Pol)(In) SAR denoising," IEEE Transactions on Geoscience and Remote Sensing, vol. 53, no. 4, pp. 2021-2038, 2015

[26] G. Gilboa, N. Sochen, and Y. Zeevi, "Variational denoising of partly textured images by spatially varying constraints," IEEE Transactions on Image processing, vol. 15, no. 8, pp. 2281-2289, 2006.

[27] C. Sutour, C.-A. Deledalle, and J.-F. Aujol, "Adaptive Regularization of the NL-Means: Application to Image and Video Denoising," Image Processing, IEEE Transactions on, vol. 23, no. 8, pp. 3506-3521, Aug 2014.

[28] J. W. Goodman, "Some fundamental properties of speckle," JOSA, vol. 66, no. 11, pp. 1145-1150, 1976.

[29] J. Fan, M. Farmen, and I. Gijbels, "Local maximum likelihood estimation and inference," Journal of the Royal Statistical Society: Series B (Statistical Methodology), vol. 60, no. 3, pp. 591-608, 1998.

[30] J. Polzehl and V. Spokoiny, "Propagation-separation approach for local likelihood estimation," Probability Theory and Related Fields, vol. 135, no. 3 , pp. 335-362, 2006. 
[31] C.-A. Deledalle, L. Denis, and F. Tupin, "Iterative weighted maximum likelihood denoising with probabilistic patch-based weights," IEEE Transactions on Image Processing, vol. 18, no. 12, pp. 2661-2672, Dec 2009.

[32] C. Barnes, E. Shechtman, D. B. Goldman, and A. Finkelstein, "The generalized patchmatch correspondence algorithm," in European Conference on Computer Vision. Springer, 2010, pp. 29-43.

[33] J.-S. Lee, "Digital image smoothing and the sigma filter," Computer vision, graphics, and image processing, vol. 24, no. 2, pp. 255-269, 1983.

[34] J.-S. Lee, "Refined filtering of image noise using local statistics," Computer graphics and image processing, vol. 15, no. 4, pp. 380-389, 1981.

[35] C.-A. Deledalle, L. Denis, G. Poggi, F. Tupin, and L. Verdoliva, "Exploiting patch similarity for SAR image processing: the nonlocal paradigm," IEEE Signal Processing Magazine, vol. 31, no. 4, pp. 6978, 2014.

[36] F. Baselice, A. Budillon, G. Ferraioli, V. Pascazio, and G. Schirinzi, "Multibaseline SAR Interferometry from Complex Data," IEEE Journal of Selected Topics in Applied Earth Observations and Remote Sensing, vol. 7, no. 7, pp. 2911-2918, July 2014.

[37] H. Ishikawa, "Exact optimization for Markov random fields with convex priors," IEEE Transactions on Pattern Analysis and Machine Intelligence, vol. 25, no. 10, pp. 1333-1336, 2003.

[38] S. Lobry, L. Denis, and F. Tupin, "Multitemporal SAR image decomposition into strong scatterers, background, and speckle," IEEE Journal of Selected Topics in Applied Earth Observations and Remote Sensing, vol. 9, no. 8, pp. 3419-3429, 2016.

[39] A. Shabou, J. Darbon, and F. Tupin, "Multilabel partition moves for MRF optimization," Image and Vision Computing, vol. 31, no. 1, pp. 14-30, 2013.

[40] T. Pock, D. Cremers, H. Bischof, and A. Chambolle, "Global solutions of variational models with convex regularization," SIAM Journal on Imaging Sciences, vol. 3, no. 4, pp. 1122-1145, 2010.

[41] D. C. Ghiglia and M. D. Pritt, Two-Dimensional Phase Unwrapping: Theory, Algorithms, and Software, Wiley, 1998.

[42] J. Bioucas-Dias and G. Valadao, "Phase unwrapping via graph cuts," Image Processing, IEEE Transactions on, vol. 16, no. 3, pp. 698-709, 2007.

[43] G. Ferraioli, G. Ferraiuolo, and V. Pascazio, "Phase-offset estimation in multichannel sar interferometry," IEEE Geoscience and Remote Sensing Letters, vol. 5, no. 3, pp. 458-462, 2008.

[44] A. Shabou, , and F. Tupin, "A Markovian Approach for DEM Estimation From Multiple InSAR Data With Atmospheric Contributions," IEEE Geoscience and Remote Sensing Letters, vol. 9, no. 4, pp. 764-768, 2012.

[45] C. Dubois, A. Thiele, and S. Hinz, "Building detection and building parameter retrieval in InSAR phase images," ISPRS Journal of Photogrammetry and Remote Sensing, vol. 114, pp. "228 - 241, 2016.

[46] C. Rossi and M. Eineder, "High-Resolution InSAR Building Layovers Detection and Exploitation," IEEE Transactions on Geoscience and Remote Sensing, vol. 53, no. 12, pp. 6457-6468, 2015. 